\title{
A Brief Study of the Universe
}

\author{
Subir Kumar Basu Thakur, Sudeshna Mukerji* \\ Department of Mathematics, Jadavpur University, Kolkata, India \\ Email: "mukerjisudeshna@gmail.com
}

Received January 22, 2013; revised February 24, 2013; accepted March 4, 2013

Copyright (C) 2013 Subir Kumar Basu Thakur, Sudeshna Mukerji. This is an open access article distributed under the Creative Commons Attribution License, which permits unrestricted use, distribution, and reproduction in any medium, provided the original work is properly cited.

\begin{abstract}
In the present study, an oscillatory model of the universe is proposed wherein the universe undergoes a sequence of big bang, expansion, contraction, big bang-repeated ad infinitum. The universe comprises of a "World” and an “Antiworld" in both of which matter operates in the positive time zone and antimatter in the negative time zone. Big bang is predicted as the violent encounter between matter and antimatter. It is suggested that antimatter has negative mass and is hugely abundant. It is also shown why it is extremely rare in spite of its abundance. It is predicted that a built in transformation converts matter to antimatter and vice versa. Finally, it is established that symmetry between matter and antimatter in the universe is maintained throughout.
\end{abstract}

Keywords: Cosmology

\section{Introduction}

\subsection{Methodology}

This paper presents a study of the universe using a new method, to be named as the "Method of Indices". It is named so, because it defines and evaluates the "Growth Indices” (briefly, "Indices”) of scalars, at play in the universe, links these indices to the Hubble's Constant, and subjects various equations of Physics and Cosmology to unveil new cosmological truths.

The index of any scalar, $X$, appearing in an equation of physics or cosmology, is denoted by $X^{\prime}$ and given by

$$
X^{\prime}=\frac{\dot{X}}{|X|}
$$

where $\dot{X}=\frac{\mathrm{d} X}{\mathrm{~d} t}$, $t$ denoting time. $|X|$ in the denominator ensures that $X^{\prime}$ has the same sign as that of $\dot{X}$.

So, $X^{\prime}>0$ and $X^{\prime}<0$ indicate respectively the growth and decay of $X$ with advancing time. $X^{\prime}=0$ marks $X$ as a universal constant, a constant over the eons.

The method is now described briefly. Using rules, given in Section 1.3, of operations involving indices, an equation of physics or cosmology can be transformed

\footnotetext{
"Corresponding author.
}

into a linear equation in the indices of scalars, which constitute the former equation. Any carefully chosen set of such linear equations, with at least one among them involving the Hubble's constant, is solvable for the unknown indices. Indices, thus obtained, reflect the effect only of the Hubble's expansion. Each of these indices promotes an equation, which links the corresponding scalar to time. The promoted equation will enable scientists to evaluate the scalar for any chosen time, provided its value for any other time is known.

Next, the known constants of physics and cosmology are constants for the present epoch, but may or may not be so over the eons. So, a prerequisite for the application of the method of indices is set up as follows.

"Each scalar except the velocity of light $(c)$, be it a constant or not, is to be regarded from the outset of the analysis as varying with time till the analysis marks it as a constant for all eons. For a scalar $X$ to be so, $X^{\prime}$ must be zero."

Though the Hubble's law plays a pivotal role in this method, it itself is subjected to analysis by this method. And for this, $H_{0}$, the Hubble's Constant of this law, is replaced by $H$, which is considered as varying with time, $H_{0}$ being the value of $H$ at the present epoch.

\subsection{Definitions and Notations}

1) A spatial sphere will be referred to as a "3-sphere". 
2) $S$ will denote the radius of the three sphere of the universe.

3) $M$ and $m$ will denote the mass of matter and antimatter respectively.

4) The generalised scalar, denoted by $X$, will represent all scalars individually, when such situation arises.

5) The constants of integration in all equations will be denoted by $A$ without suffices and must not be considered as equal dimensionally or otherwise.

6) Scalars at $t=0$ and $t=t_{0}$ (present epoch) will have suffices " $\mathrm{i}$ " and " 0 " respectively.

7)

$$
c^{\prime}=0
$$

8) The Hubble's Law states that galaxies move away from an observer with speeds $\dot{R}$, directly proportional to their respective distances $R$ from the observer i.e.

$$
\frac{\dot{R}}{R}=H_{0}
$$

where $H_{0}$ i.e. the Hubble's Constant. Now, as $R>0 \Rightarrow R=|R|$ and $H_{0}$ is to be replaced by $H$ (as proposed in Section 1.0.1), then $\frac{\dot{R}}{|R|}=H$ is the Hubble's Law which can now be written in the following new form:

$$
R^{\prime}=H
$$

9) In all numerical calculations, following values of the Gravitational Constant (G), the Hubble's Constant $(\mathrm{H})$ and the velocity $(c)$ of light for the present epoch will be used

$$
\begin{gathered}
G_{0}=6.672 \times 10^{-11} \mathrm{Nm}^{2} \cdot \mathrm{Kg}^{-2} \\
H_{0}^{-1}=13.7 \times 10^{-9} \text { yrs. }=4.320432 \times 10^{17} \mathrm{~s} \\
c=2.99792458 \times 10^{8} \mathrm{~m} \cdot \mathrm{s}^{-1}
\end{gathered}
$$

\subsection{Rules of Operations for the Indices}

Rules of operations for indices of two positive scalars, $A$ and $B$, with respect to positive time, are provided below. Whenever used, $a$ will denote a positive real number. The rules can be extended to any number of positive scalars. Higher order indices also exist, an example will be provided in Subsection 4.2.

1) If $A$ and $B$ are of the same type,

$$
A^{\prime}=B^{\prime}
$$

But, $\quad c^{\prime} \neq v^{\prime}$

2)

$$
(a A)^{\prime}=A^{\prime}
$$

3)

$$
(-a A)^{\prime}=-A^{\prime}
$$

4)

$$
\left(A^{a}\right)^{\prime}=a A^{\prime}
$$

5)

$$
\left(A^{-a}\right)^{\prime}=-a A^{\prime}
$$

6)

$$
\left(-A^{a}\right)^{\prime}=-a A^{\prime}
$$

7)

$$
\left(A^{-a}\right)^{\prime}=\left(-A^{a}\right)^{\prime}=-a A^{\prime}
$$

8)

$$
\left(A^{-1}\right)^{\prime}=(-A)^{\prime}=-A^{\prime}
$$

9)

$$
(A B)^{\prime}=A^{\prime}+B^{\prime}
$$

10)

$$
\left(\frac{A}{B}\right)=A^{\prime}-B^{\prime}
$$

11)

$$
(A+B)^{\prime}=\left(\frac{|A|}{|A+B|}\right) A^{\prime}+\left(\frac{|B|}{|A+B|}\right) B^{\prime}
$$

12) For $A>B$,

$$
(A-B)^{\prime}=\left(\frac{|A|}{|A-B|}\right) A^{\prime}-\left(\frac{|B|}{|A-B|}\right) B^{\prime}
$$

\section{Analysis}

\subsection{Hubble's Law}

Since $R$ and $S$ are of the same type, both being lengths, by rule 1 of Subsection 1.3, Equation (4) is written as [1]

$$
H=S^{\prime}
$$
$H=S^{\prime}$
As $\quad S \geq 0, \quad|S|=S ;$ and so, $S^{\prime}=\frac{\dot{S}}{S}$, whence
Equation (17) becomes

$$
H=\frac{\dot{S}}{S}
$$

So,

$$
\dot{H}=\frac{\ddot{S}}{S}-H^{2}
$$


If

$$
\frac{\ddot{S}}{S}=-q H^{2}
$$

then

$$
\dot{H}=-(1+q) H^{2}
$$

$q_{0}$, the value of $q$ at the present epoch, is the 'deceleration parameter'. As $H>0, H=|H|$, and so by Equation (20), $\frac{\dot{H}}{|H|}=-(1+q) H$, whence

$$
H^{\prime}=-(1+q) H
$$

Next, as the universe is expanding, $\dot{S}>0$, and so,

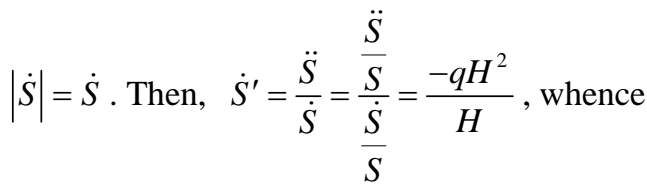

$$
\dot{S}^{\prime}=q H
$$

Also, $t^{\prime}=\frac{\frac{\mathrm{d} t}{\mathrm{~d} t}}{|t|}$,

$$
t^{\prime}=\frac{1}{t}
$$

\subsection{Friedmann's Equations}

The Friedmann's equations for $k=0$ complete with the "Cosmological Constant" $(\Lambda)$, are [2]

$$
\begin{gathered}
(1-2 q) H^{2}=\Lambda c^{2} \\
H^{2}-\frac{\Lambda c^{2}}{3}=\frac{8 \pi G \bar{\rho}_{0} S_{0}^{3}}{3 S^{3}}
\end{gathered}
$$

where $\bar{\rho}_{0}$ is the rest mass density of the universe st $t=t_{0}$. Let

$$
\Lambda=\frac{\sigma^{2}}{S_{0}^{2}}
$$

where $\sigma$ is a parameter, that sets the level of $\Lambda$. Then,

$$
\Lambda c^{2}=\frac{\sigma^{2} c^{2}}{S_{0}^{2}}=\frac{\sigma^{2}}{t_{0}^{2}}
$$

So, the Friedmann's equations are rewritten as:

$$
\begin{aligned}
& (1-2 q) H^{2}=\frac{\sigma^{2}}{t_{0}^{2}} \\
& H^{2}-\frac{\sigma^{2}}{3 t_{0}^{2}}=\frac{8 \pi G \bar{\rho}_{0} S_{0}^{3}}{3 S^{3}}
\end{aligned}
$$

\subsection{The Nature of $q$}

Differentiation of Equation (28) with respect to time yields:

$$
\dot{q}=(1-2 q) H^{\prime}
$$

Then, by Equation (21) this becomes:

$$
\dot{q}=-(1-2 q)(1+q) H
$$

So,

$$
q^{\prime}=\frac{-(1-2 q)(1+q) H}{|q|}
$$

$q^{\prime}$ is non-zero except for $q=\frac{1}{2}$ and $q=-1$. So, $q$ varies with time; in fact, it decreases from $q=\frac{1}{2}$ to $q=-1$.

\subsection{More about the Friedmann's Equations}

Let two quantities, $\lambda$ and $\Omega$, be introduced below [2]:

$$
\begin{array}{r}
\lambda=\frac{\Lambda c^{2}}{3 H^{2}}=\frac{\sigma^{2} c^{2}}{3 S_{0}^{2} H^{2}}=\frac{\sigma^{2}}{3 t_{0}^{2} H^{2}} \\
\Omega=\frac{8 \pi G \bar{\rho}_{0} S_{0}^{3}}{3 H^{2} S^{3}}=\frac{8 \pi G \bar{\rho}}{3 H^{2}}
\end{array}
$$

It will be proved in this very subsection that they vary with time. Their values, $\lambda_{0}$ and $\Omega_{0}$, at $t=t_{0}$ are the well known "Cosmological Constant Parameter" $\left(\Omega_{\Lambda}\right)$ and "Density Parameter" $\left(\Omega_{0}\right)$ respectively.

Equation (28) implies: $\frac{\sigma^{2}}{3 t_{0}^{2} H^{2}}=\frac{1-2 q}{3}$, whence

$$
\lambda=\frac{1-2 q}{3}
$$

Next, Equation (29) implies: $1-\frac{\sigma^{2}}{3 t_{0}^{2} H^{2}}=\frac{8 \pi G \bar{\rho}}{3 H^{2}}$, whence

$$
\lambda+\Omega=1
$$

Equations (35) and (36) together imply:

$$
\Omega=\frac{2(1+q)}{3}
$$

Also, Equations (35) and (37) together imply:

$$
\Omega-2 \lambda=2 q
$$

Now, $S=c t \Rightarrow S^{\prime}=c^{\prime}+t^{\prime} \Rightarrow S^{\prime}=t^{\prime}$ by Equation (2). Since by Equation (23), $H=\frac{1}{t}$, then

$$
H t=1
$$


Next, by Equation (39), $\frac{C}{S}=\frac{1}{t} \Rightarrow \frac{C}{S}=H$. Then,

$$
\frac{c}{S H}=1
$$

Then,

$$
\frac{c^{2}}{S_{0}^{2} H_{0}^{2}}=1
$$

Equations (33) and (41) together imply:

$$
\sigma^{2}=3 \lambda_{0}
$$

By Equation (35) this becomes:

$$
\sigma=\sqrt{1-2 q_{0}}
$$

Equations (35) and (37) respectively show that $\lambda$ and $\Omega$ vary with $q$, which has been shown in Section 2.3 to be varying with time. So, $\lambda$ and $\Omega$ also vary with time.

In fact, at $t=0$, when $q=\frac{1}{2}, \lambda=0$ and $\Omega=1$. And, when $q=-1, \lambda=1$ and $\Omega=0 . \Omega=0$ means the universe is empty. So, the condition that the universe is empty is $\Omega=0$, or $\lambda=1$, or $q=-1$.

In de Sitter's universe, $\Lambda=\frac{3 H^{2}}{c^{2}} \Rightarrow \frac{\Lambda c^{2}}{3 H^{2}}=1$. So, by Equation (33), $\lambda=1$. So, de Sitter's universe is empty.

\section{5. $q$ as a Function of Time}

Equation (31) is written as:

$$
\dot{q}=-(1+q) \sqrt{1-2 q}(H \sqrt{1-2 q})
$$

By Equation (28) this becomes:

$$
\begin{aligned}
\frac{\dot{q}}{\sqrt{1-2 q}}= & -(1+q)\left(\frac{\sigma}{t_{0}}\right) \Rightarrow \frac{-\mathrm{d} q}{\sqrt{1-2 q}}=\frac{(3-(1-2 q))}{2} \frac{\sigma}{t_{0}} \mathrm{~d} t \\
& \Rightarrow \frac{\mathrm{d}(\sqrt{1-2 q})}{\left[(\sqrt{3})^{2}-(\sqrt{1-2 q})^{2}\right]}=\frac{\sigma}{2 t_{0}} \mathrm{~d} t
\end{aligned}
$$

Integration of this yields:

$$
\frac{1}{2 \sqrt{3}} \ln \left|\frac{\sqrt{3}+\sqrt{1-2 q}}{\sqrt{3}-\sqrt{1-2 q}}\right|=\frac{\sigma t}{2 t_{0}}+A
$$

where $A$ is the constant of integration.

Now,

$$
\frac{1}{2} \geq q \geq-1 \Rightarrow 0 \leq 1-2 q \leq 3 \Rightarrow \sqrt{3}-\sqrt{1-2 q} \geq 0
$$

So, $\frac{\sqrt{3}+\sqrt{1-2 q}}{\sqrt{3}-\sqrt{1-2 q}} \geq 0$; then,

$$
\left|\frac{\sqrt{3}+\sqrt{1-2 q}}{\sqrt{3}-\sqrt{1-2 q}}\right|=\frac{\sqrt{3}+\sqrt{1-2 q}}{\sqrt{3}-\sqrt{1-2 q}}
$$

So,

$$
\ln \frac{\sqrt{3}+\sqrt{1-2 q}}{\sqrt{3}-\sqrt{1-2 q}}=\frac{\sqrt{3} \sigma t}{t_{0}}+2 \sqrt{3} A
$$

At $t=0 \quad q=\frac{1}{2}$; so, $A=0$. Then,

$$
\frac{\sqrt{3}+\sqrt{1-2 q}}{\sqrt{3}-\sqrt{1-2 q}}=\mathrm{e}^{\frac{\sqrt{3} \sigma t}{t_{0}}}
$$

or,

$$
\left(\mathrm{e}^{\frac{\sqrt{3} \sigma t}{t_{0}}}+1\right) \sqrt{1-2 q}=\sqrt{3}\left(\mathrm{e}^{\frac{\sqrt{3} \sigma t}{t_{0}}}-1\right)
$$

whence

$$
1-2 q=\frac{3\left(\mathrm{e}^{\frac{\sqrt{3} \sigma t}{t_{0}}}-1\right)^{2}}{\left(\mathrm{e}^{\frac{\sqrt{3} \sigma t}{t_{0}}}+1\right)^{2}}
$$

Therefore,

$$
q=\frac{1}{2}-\frac{3}{2} \frac{\left(\mathrm{e}^{\frac{\sqrt{3} \sigma t}{t_{0}}}-1\right)^{2}}{\left(\mathrm{e}^{\frac{\sqrt{3} \sigma t}{t_{0}}}+1\right)^{2}}
$$

\subsection{Evaluation of $\sigma$ and $q_{0}$}

At $t=t_{0}$, Equation (45) becomes:

$$
1-2 q_{0}=\frac{3\left(\mathrm{e}^{\sqrt{3} \sigma}-1\right)^{2}}{\left(\mathrm{e}^{\sqrt{3} \sigma}+1\right)^{2}}
$$

By Equation (43), this further changes to:

$$
\sigma^{2}=\frac{3\left(\mathrm{e}^{\sqrt{3} \sigma}-1\right)^{2}}{\left(\mathrm{e}^{\sqrt{3} \sigma}+1\right)^{2}}
$$

This equation is satisfied by:

$$
\sigma^{2}=2.211373949
$$

Then, by Equation (43),

$$
q_{0}=-0.605686974
$$




\subsection{Evaluation of $t_{0}$ and $S_{0}$}

$$
\begin{gathered}
t_{0}=H_{0}^{-1}=13.7 \times 10^{9} \text { yrs. }=4.320432 \times 10^{17} \mathrm{~s} . \\
S_{0}=c t_{0}=12.95232929 \times 10^{25} \mathrm{~m} .
\end{gathered}
$$

\subsection{Indices of Few Scalars from Equations of Physics}

1) Index of mass of matter: Angular momentum of a body of mass $M$, revolving in a circular orbit of radius $r$ with linear velocity $v$ in a direction tangential to the orbit, is constant; i.e. $M v r=\gamma$, where $\gamma$ is the constant angular momentum. Using Equation (13) this is transformed to: $M^{\prime}+v^{\prime}+r^{\prime}=0$

By Equations (5) and (22), $v^{\prime}=-q H$, and by Equations (5) and (17), $r^{\prime}=H$. So, $M^{\prime}-q H+H=0$

$$
M^{\prime}=-(1-q) H
$$

2) Index of force: The centripetal force, acting on the revolving body, referred to in above, is $F=\frac{m v^{2}}{r}$.

Using Equations (3), (8), and (14) to this :

$$
F^{\prime}=M^{\prime}+2 v^{\prime}-r^{\prime}=-(1-q) H-2 q H-H
$$

whence

$$
F^{\prime}=-(2+q) H
$$

3) Index of gravitational constant $(G)$ : The gravitational force of attraction between two bodies of mass $M_{1}$ and $M_{2}$ at a distance $R$ is:

$$
F=\frac{G M_{1} M_{2}}{R^{2}} \text {, whence } G=\frac{F R^{2}}{M_{1} M_{2}} \text {. }
$$

Then, $G^{\prime}=F^{\prime}+2 R^{\prime}-2 M^{\prime}$; or, $G^{\prime}=-(2+q) H+2 H+2(1-q) H$, whence

$$
G^{\prime}=(2-3 q) H
$$

\subsection{A Few More Indices}

1) Index of $\beta_{R}\left(=\frac{\dot{R}}{C}\right)$ for $0<R<S$ : For any $R(0<R<S)$,

$$
\begin{gathered}
\dot{R}=H R \Rightarrow \frac{\dot{R}}{c}=\left(\frac{H}{c}\right) R \Rightarrow \beta_{R}=\frac{R}{S} \\
\Rightarrow \beta_{R}^{\prime}=R^{\prime}-S^{\prime} \Rightarrow \beta_{R}^{\prime}=H-H .
\end{gathered}
$$

Then,

$$
\beta_{R}^{\prime}=0
$$

2) Index of $\sqrt{1-\beta_{R}^{2}}$ : By Equation (16), as $\beta_{R}<1$,

$$
\left(1-\beta_{R}^{2}\right)^{\prime}=-\left(\frac{\left|\beta_{R}^{2}\right|}{\left|1-\beta_{R}^{2}\right|}\right)\left(\beta_{R}^{2}\right)^{\prime}=-\left(\frac{\left|\beta_{R}^{2}\right|}{\left|1-\beta_{R}^{2}\right|}\right)\left(2 \beta_{R}^{\prime}\right) .
$$

Then, by Equation (54), $\left(1-\beta_{R}^{2}\right)^{\prime}=0$. Now, $\left(\sqrt{1-\beta_{R}^{2}}\right)=\frac{1}{2}\left(1-\beta_{R}^{2}\right)^{\prime}$, whence

$$
\left(\sqrt{1-\beta_{R}^{2}}\right)=0
$$

3) Index of $\Omega: \Omega^{\prime}=\left(\frac{2(1+q)}{3}\right) \Rightarrow \Omega^{\prime}=(1+q)^{\prime}$ (by Equation (6)) $\Rightarrow \Omega^{\prime}=\frac{\frac{\mathrm{d}(1+q)}{\mathrm{d} t}}{|1+q|}$.

As $q>-1,1+q>0$, and so, $|1+q|=1+q$. Then, $\Omega^{\prime}=\frac{\dot{q}}{1+q}$. So, by Equation (31),

$$
\Omega^{\prime}=-(1-2 q) H
$$

4) Index of $\lambda: \lambda^{\prime}=\left(\frac{(1-2 q)}{3}\right)$. By Equation (6), $\lambda^{\prime}=(1-2 q)^{\prime}$. Then, $\lambda^{\prime}=\frac{\frac{\mathrm{d}(1-2 q)}{\mathrm{d} t}}{|1-2 q|}=\frac{-2 \dot{q}}{1-2 q}$, as $(1-2 q) \geq 0$. Then, by Equation (31),

$$
\lambda^{\prime}=2(1+q) H
$$

\subsection{Why Does the Universe Expand?}

That the mass of matter in the universe is decreasing with advancing time is evident from the negative value of $M^{\prime}$, obtained in Equation (51). In this decreasing process, $M^{\prime}$ is the index of the residual mass, and $\left(-M^{\prime}\right)$ is that of the withdrawn mass (mass that ceases to be so). Equation (51) implies: $-M^{\prime}=S^{\prime}+\dot{S}^{\prime}$. This proves that the withdrawn mass creates vacuum energy that promotes the accelerative expansion $\left(S^{\prime}+\dot{S}^{\prime}\right)$ of the universe. Next, Equation (34) can be written as:

$$
\Omega=\frac{\bar{M}}{\frac{H^{2} S^{3}}{2 G}}
$$

where $\bar{M}$ is the residual mass of the universe. The denominator has the dimension of mass. So, $\Omega$ is the proportion of $\bar{M}$ to $\frac{H^{2} S^{3}}{2 G}$, which represents the total rest mass (in nature). Then, the withdrawn rest mass is 
$\left(\frac{H^{2} S^{3}}{2 G}\right)-\bar{M}$. So, the proportion of the withdrawn rest mass is:

$$
1-\frac{\bar{M}}{\frac{H^{2} S^{3}}{2 G}}=1-\Omega=\lambda .
$$

This shows that another measure of the withdrawn mass is $\lambda$, which is regarded as the driving factor behind the accelerative expansion of the universe.

Thus, the accelerative expansion of the universe is due to the vacuum energy created by the withdrawing mass, indicated by $\left(-M^{\prime}\right)$ and $\lambda$.

\section{Antimatter in the Universe}

\subsection{Why Antimatter?}

Antimatter is no less a reality than matter [3,4]. This fact alone is reason enough to undertake an investigation into the possibility of antimatter, playing a role, similar to that played by matter, in the workings of the universe. It's extreme rarity has so long relegated it to insignificance.

It will be proved in Subsection 3.3 that antimatter is hugely abundant. It will also be explained, in the two subsequent subsections, why, in spite of its abundance, it is rare.

\subsection{Dynamic Relation between $M$ and $|m|$}

If $M$ is the mass of a number of particles, then the mass $(m)$ of the same number of antiparticles is given by [5-7]:

$$
m=-M
$$

This implies

$$
m^{\prime}=-M^{\prime} \Rightarrow m^{\prime}+M^{\prime}=0 \Rightarrow(m M)^{\prime}=0 \Rightarrow m M=-\theta,
$$

where $\theta(>0)$ is a constant. Then, $-m M=\theta$, whence

$$
M|m|=\theta
$$

This is the dynamic relation between particles and antiparticles. This is called so, because the ratio $\left(\frac{|m|}{M}\right)$ varies with time, as shown below.

$$
M|m|=\theta \Rightarrow \frac{|m|}{M}=\frac{\theta}{M^{2}} \Rightarrow\left(\frac{|m|}{M}\right)=\left(\frac{\theta}{M^{2}}\right)
$$

Then, $\left(\frac{|m|}{M}\right)=\left(M^{-2}\right)^{\prime}=-2 M^{\prime} \neq 0$.

\subsection{Abundance of Antimatter}

Friedmann's Equation (34) has been rewritten in Equa- tion (58), which is now changed below to it's equivalent equation in indices.

$$
\Omega^{\prime}=G^{\prime}+M^{\prime}-2 H^{\prime}-3 S^{\prime}
$$

As $\bar{M}$ is the rest mass of the universe, it is given by:

$$
\bar{M}=M \sqrt{1-\beta_{R}^{2}},
$$

where $M$ is the mass of the universe, and $\beta_{R}=\frac{\dot{R}}{C}$, $\dot{R}$ being the average speed of mass. Now, $\dot{R}=R H$, and $0<\dot{R}<\dot{S}$; so, $0<R<S$. For such $R$, and consequently for $\dot{R},\left(\sqrt{1-\beta_{R}^{2}}\right)$ has been evaluated in Equation (55) as $\left(\sqrt{1-\beta_{R}^{2}}\right)=0$. Then

$$
\bar{M}^{\prime}=M^{\prime}
$$

Equation (61) can be written as:

$$
M^{\prime}=\Omega^{\prime}+2 H^{\prime}+3 S^{\prime}-G^{\prime}
$$

By Equations (17), (21), (53), and (56), the above equation becomes:

$$
M^{\prime}=-(2-3 q) H
$$

This does not agree with Equation (51), which gives $M^{\prime}=-(1-q) H$. So, $M$ of Equation (64) must have a component other than the mass of matter. There can be no such component other than the mass of antimatter. This prompts replacement of $M$ by $(M+m)$, i.e., by $(M-|m|)$ in Equation (64) as given below.

$$
(M-|m|)^{\prime}=-(2-3 q) H
$$

Now, by Equation (15),

$$
\begin{aligned}
& (M-|m|)^{\prime}=\frac{|M| M^{\prime}}{|M-| m||}-\frac{|m||m|^{\prime}}{|M-| m||} \text {. } \\
& \text { Equation (60) } \Rightarrow M^{\prime}+|m|^{\prime}=0 \Rightarrow|m|^{\prime}=-M^{\prime} \text {. So, } \\
& \qquad(M-|m|)^{\prime}=\frac{(M+|m|) M^{\prime}}{|M-| m||}
\end{aligned}
$$

Since during the expansion of the universe, $M>|m|$, $|M-| m||=M-|m|$. Then,

$$
\frac{M+|m|}{M-|m|}=\frac{(M-|m|)^{\prime}}{M^{\prime}}
$$

So, by Equations (65) and (51), we have

$$
\frac{M+|m|}{M-|m|}=\frac{-(2-3 q) H}{-(1-q) H}
$$

Then, at $t=t_{0}$, 


$$
\frac{M_{0}+\left|m_{0}\right|}{M_{0}-\left|m_{0}\right|}=\frac{2-3 q_{0}}{1-q_{0}}
$$

This, with Equation (48) gives

$$
\frac{\left|m_{0}\right|}{M_{0}}=0.407795824
$$

Now, at any time $t=t$, let a sphere concentric with the 3-sphere of the universe is of radius $R(0 \leq R \leq S)$ and have the density $\rho_{R}$. Then the mass of this sphere is $M_{R}=a \rho_{R} R^{3}$, where $a=\frac{4 \pi}{3}$.

Let another concentric sphere of radius $R+d R$ be considered. The density of this sphere can, for all practical purposes, be taken as $\rho_{R}$, since $d R$ is infinitesimally small. Then, $M_{R}+d M_{R}$, the mass of this sphere is: $a \rho_{R}(R+d R)^{3}=a \rho_{R}\left(R^{3}+3 R^{2} d R\right)$, neglecting higher powers of $d R$ than one. So, $d M_{R}=3 a \rho_{R} R^{2} d R$.

Then, the average speed of the entire mass of the 3-sphere of the universe is:

$$
\dot{R}=\frac{\int_{0}^{M}(R H) \mathrm{d} M_{R}}{\int_{0}^{M} \mathrm{~d} M_{R}}
$$

where $R H$ is the speed of the galaxies at a distance $R$ from the observer, or for all practical purposes, the speed of matter within the spherical shell, having the outer and inner radii $R+d R$ and $R$ respectively. so,

$$
\dot{R}=\frac{3 a \rho_{R} H \int_{0}^{S} R^{3} \mathrm{~d} R}{3 a \rho_{R} \int_{0}^{S} R^{2} \mathrm{~d} R}=\frac{\frac{H S^{4}}{4}}{\frac{S^{3}}{3}}=\frac{3 H S}{4}
$$

So, by Equation (40), $\dot{R}=\frac{3 c}{4}$, whence

$$
\beta_{R}=\frac{3}{4}
$$

Therefore,

$$
\sqrt{1-\beta_{R}^{2}}=\frac{\sqrt{7}}{4}
$$

Now, Equation (58) implies: $\bar{M}=\frac{\Omega H^{2} S^{3}}{2 G}$

As $\bar{M}=M \sqrt{1-\beta_{R}^{2}}$, by Equation (68), the above becomes:

$$
M=\left(\frac{4}{\sqrt{7}}\right) \frac{\Omega H^{2} S^{3}}{2 G}
$$

Replacement of $M$ by $M-|m|$ changes this to:

$$
M-|m|=\left(\frac{2}{\sqrt{7}}\right) \frac{\Omega H^{2} S^{3}}{G}
$$

At $t=t_{0}$, this becomes:

$$
M_{0}-\left|m_{0}\right|=\left(\frac{2}{\sqrt{7}}\right) \frac{\Omega_{0} H_{0}^{2} S_{0}^{3}}{G_{0}}
$$

Equations (37) and (48) together give $\Omega_{0}=0.26287535$. Values of $H_{0}$ and $G_{0}$ are taken from Subsection 1.2 and $S_{0}$ from Equation (50). With these values, Equation (70) gives

$$
M_{0}-\left|m_{0}\right|=3.467081744 \times 10^{52} \mathrm{Kg}
$$

From this with the help of Equation (66), $M_{0}$ and $\left|m_{0}\right|$ are evaluated as given below:

$$
\begin{gathered}
M_{0}=5.854537868 \times 10^{52} \mathrm{Kg} \\
\left|m_{0}\right|=2.387456094 \times 10^{52} \mathrm{Kg}
\end{gathered}
$$

\subsection{Static Relation between $M$ and $|m|$}

The static relation between $M$ and $|m|$ is characterised by $\left(\frac{|m|}{M}\right)^{\prime}=0$. This relation is obtained as follows: Equation (59) $\Rightarrow M=-m$, whence

$$
M=|m|
$$

This is the static relation. Then,

$$
M^{\prime}=|m|^{\prime}
$$

This implies $|m|^{\prime}-M^{\prime}=0 \Rightarrow\left(\frac{|m|}{M}\right)^{\prime}=0$. Thus the criterion of static relation is fulfilled by Equation (73). Next, Equation (60) $\Rightarrow M^{\prime}+|m|^{\prime}=0$, whence

$$
M^{\prime}=-|m|^{\prime}
$$

Thus Equations (74) and (75) do not agree, seemingly posing a problem. It will be seen in Subsection 3.7 that the dynamism of relation (75) originating from the static relation (74) is the solution to this problem.

\subsection{Two Time Zones}

The concept of two time zones is necessary to show that the dynamism of relation (75) originates from the static relation (74). This concept is there in the theory of Quantum Electrodynamics also. This theory will come up again in Subsection 3.7.

This concept necessitates introducing a new convention of denoting the time derivative and the indices of scalars as is given below. Suffices " $t$ " (or, "+") and "- $t$ " (or, "-") will be used under this convention.

1) $\frac{\mathrm{d} X}{\mathrm{~d} t}$ and $\frac{\mathrm{d} X}{\mathrm{~d}(-t)}$ will respectively be denoted by 
$\dot{X}_{t}$ and $\dot{X}_{-t}$.

2) $\frac{\dot{X}_{t}}{|X|}$ and $\frac{\dot{X}_{-t}}{|X|}$ will respectively be denoted by $X_{t}^{\prime}$ and $X_{-t}^{\prime}$.

Also, two relations between $X_{t}^{\prime}$ and $X_{-t}^{\prime}$ are deduced below.

a)

$$
-X_{t}^{\prime}=\frac{-\frac{\mathrm{d} X}{\mathrm{~d} t}}{|X|}=\frac{\frac{\mathrm{d} X}{\mathrm{~d}(-t)}}{|X|}
$$

whence

$$
-X_{t}^{\prime}=X_{-t}^{\prime}
$$

b) Equation (76) implies

$$
X_{t}^{\prime}=-X_{-t}^{\prime}
$$

It must be mentioned here that all indices, occurring upto Subsection 3.4, are of the type $X_{t}^{\prime}$.

Now, the static relation (73) $\Rightarrow M_{t}^{\prime}=|m|_{t}^{\prime}=-m_{t}^{\prime}$, whence by Equation (76),

$$
M_{t}^{\prime}=m_{-t}^{\prime}
$$

This is the fundamental relation between matter and antimatter. Also, as the natural time zone for matter is the Positive Time Zone (PTZ), Equation (78) shows that the same for antimatter is the Negative Time Zone (NTZ). Time is progressive in the PTZ and regressive in the NTZ.

Next, relation (73)

$\Rightarrow M_{t}^{\prime}=|m|_{t}^{\prime} \Rightarrow M_{t}^{\prime}=-m_{t}^{\prime} \Rightarrow m_{t}^{\prime}=-M_{t}^{\prime}$, whence by Equation (76)

$$
m_{t}^{\prime}=M_{-t}^{\prime}
$$

\subsection{The Time Barrier}

The PTZ is defined as

$$
0 \leq t \leq \infty
$$

and the NTZ is defined as

$$
0 \geq-t \geq-\infty
$$

So, matter and antimatter, when in the PTZ, moves forward in time and when they are in the NTZ, they move backward in time. The only instant of time common to both PTZ and NTZ is:

$$
t=-t=0
$$

Since matter and antimatter are trapped in the PTZ and the NTZ respectively when Equation (78) rules, matter cannot cross over to the NTZ as matter, and antimatter cannot cross over to the PTZ antimatter, and so there exists a "time barrier" (except $t=-t=0$ ) to prevent matter and antimatter from meeting. So, antimatter cannot be sighted in the PTZ, when Equation (78) rules.

When Equation (79) rules, antimatter and matter appear in the PTZ and NTZ, not in the NTZ and PTZ respectively. Since they appear in their unusual time zones, their appearances must be extremely rare. So, extremely rare are the appearances of antimatter in the PTZ. Such rare appearances of antimatter result from chance phenomena like cosmic rays striking interstellar matter.

\subsection{Conversion}

Equation (78) is the result of the following transformation:

$$
(t, M) \rightarrow(-t, m)
$$

This transformation means that matter in the PTZ gets converted to antimatter in the NTZ. So, antimatter increases at the cost of matter, that decreases [vide Equation (51)]. This is exactly what the dynamic relation (60) conveys. As transformation (82) originates from the static relation, the problem, that was faced in Subsection 3.4, vanishes.

Next, Equation (79) is the result of the following transformation

$$
(t, m) \rightarrow(-t, M)
$$

This transformation shows that antimatter, which is in the PTZ and so moves forward in time, gets converted to matter, which is in NTZ and so moves backward in time [8]. In the theory of Quantum Electrodynamics also, antimatter moving forward in time is interpreted as matter moving backward in time.

\subsection{Rarity of Antimatter}

When Equation (78) rules, antimatter is hugely abundant in the NTZ at present, and because of the time barrier there is absolutely no chance of antimatter appearing in the PTZ and being detected. But when Equation (79) rules, antimatter appears in the PTZ. It has been explained in Subsection 3.6 that such appearances are extremely rare. Thus it is explained why antimatter is extremely rare in spite of its huge abundance.

\section{Game Matter and Antimatter Play}

\subsection{The Three Fundamental Scalars}

The three fundamental scalars (time, radius of the 3sphere and mass), associated with antimatter, are related to the corresponding scalars, associated with matter, as given below.

$$
\begin{aligned}
& t_{a}=-t \\
& S_{a}=S
\end{aligned}
$$




$$
m=-M
$$

where the suffix “a” indicates association with antimatter.

\subsection{Matter in the Expanding Universe}

Equation (39) $\Rightarrow H=\frac{1}{t}$; so, by Equation (23),

$$
H=t_{t}^{\prime}
$$

where

$$
H=S_{t}^{\prime}
$$

Equation (39) also implies:

$$
H_{t}^{\prime}=-t_{t}^{\prime}
$$

Now, Equation (12) $\Rightarrow\left(M^{-1}\right)_{t}^{\prime}=-M_{t}^{\prime}$

So, by Equation (51),

$$
\left(M^{-1}\right)_{t}^{\prime}=(1-q) H=-(1+q) H+2 H=H_{t}^{\prime}+2 t_{t}^{\prime}=t_{t}^{\prime}
$$

whence

$$
t \propto M^{-1}
$$

Next, Equations (87) and (88) together imply:

$$
t_{t}^{\prime}=S_{t}^{\prime}
$$

whence

$$
t \propto S
$$

By Equations (90) and (91)

$$
t \propto \frac{S}{M}
$$

\subsection{Antimatter in the Expanding Universe (Figure 1)}

Equation (76) $\Rightarrow-M_{t}^{\prime}=M_{-t}^{\prime} \Rightarrow-M_{t}^{\prime}=(-m)_{-t}^{\prime}$ whence

$$
-M_{t}^{\prime}=|m|_{-t}^{\prime}
$$

Equation (92) $\Rightarrow t_{t}^{\prime}=S_{t}^{\prime}-M_{t}^{\prime}$. So, by Equation (93), $t_{t}^{\prime}=S_{t}^{\prime}+|m|_{-t}^{\prime}$.

Then, by Equation (77), this becomes:

$$
t_{t}^{\prime}=S_{t}^{\prime}+|m|_{-t}^{\prime}
$$

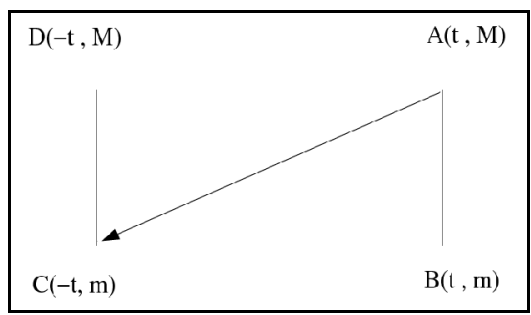

Figure 1. In the figure all the Particles in the positive time zone represented by $A(t, M)$ gets converted into the corresponding antiparticle in the negative time zone represented by $C(-t, m)$. or, $(-t)_{-t}^{\prime}=\left(S^{-1}\right)_{-t}^{\prime}+|m|_{-t}^{\prime}$, whence $-t \propto \frac{|m|}{S}$.

Now, because the LHS $<0$ and the RHS $>0$, $-t=\frac{-a|m|}{S}$, where $a(>0)$ is a constant. Then, $t=\frac{a|m|}{S}$, whence

$$
t \propto \frac{|m|}{S}
$$

\subsection{How Antimatter Increases in the Expanding Universe}

Let the four corners $A, B, C$ and $D$ of a rectangular strip of paper represent respectively the paired scalars $(t, M),(t, m),(-t, m)$ and $(-t, M)$, as shown in the figure. Then, transformations (82) and (83) can be written as below.

$$
\begin{aligned}
& A(t, M) \rightarrow C(-t, m) \\
& B(t, m) \rightarrow D(-t, M)
\end{aligned}
$$

Now, when this strip is given a single twist and the edge $A B$ is joined with the edge $C D$ in such a way that $A$ and $B$ coincide with $C$ and $D$ respectively, a Mobius strip is formed.

Then, transformations (95) and (96) together constitute a Mobius transformation operating within the universe [9]. So, under this built-in Mobius transformation in the universe, particles (in the PTZ), represented by $A(t, M)$, undergo conversion to the corresponding antiparticles (in the NTZ), represented by $C(-t, m)$. Plenty of matter in the universe ensures "continual" conversion of matter to antimatter. Such a scenario supports Equation (60) as well as the pair of Equations (92) and (94) taken together.

\subsection{Roles Played by Matter and Antimatter}

Relation (92) shows that with advancing time, decreasing matter effects the expansion of the universe, while increasing antimatter effects its contraction [vide relation (94)]. Due to matter preponderating over antimatter during the expansion phase, the contraction remains eclipsed. So, $|m|<M$ is symptomatic of the expansion of the universe. The universe will be on the threshold of inflecting into the contraction phase when

$$
|m|=M
$$

\subsection{The Moment of the Inflexion}

Let the moment of inflexion be $t=T$, and all the scalars at this moment have the suffix " $T$ ". Then, by Equation 
(97),

$\left|m_{T}\right|=M_{T}$. By Equation (60), $M_{T}\left|m_{T}\right|=M_{0}\left|m_{0}\right|$. So,

$$
M_{T}=\left|m_{T}\right|=\sqrt{M_{0}\left|m_{0}\right|}
$$

Then, by Equations (71) and (72),

$$
M_{T}=\left|m_{T}\right|=3.738643084 \times 10^{52} \mathrm{Kg}
$$

Next, by Equation (51), $M_{t}^{\prime}=-H+q H$, or,

$$
\begin{gathered}
\frac{\mathrm{d} M}{M}=(1-q)(-H \mathrm{~d} t) . \\
\text { or, } \frac{\mathrm{d} M}{M}=\frac{2 \mathrm{~d}(1+q)}{[3(1+q)]}-\frac{\mathrm{d}(1-2 q)}{[6(1-2 q)]}
\end{gathered}
$$

Integration of this gives:

$\ln |M|=\ln |1+q|^{\frac{2}{3}}+\ln |1-2 q|^{\frac{-1}{6}}+\ln A$, where $A$ is the constant of integration.

Now, since $-1 \leq q \leq \frac{1}{2},|1+q|=1+q$ and $|1-2 q|=1-2 q$. Also, $|M|=M$.

Then, $\ln M=\ln (1+q)^{\frac{2}{3}}+\ln (1-2 q)^{\frac{-1}{6}}+\ln A$; or,

$$
M=\frac{A(1+q)^{\frac{2}{3}}}{(1-2 q)^{\frac{1}{6}}}
$$

Then, $A=\frac{M_{0}\left(1-2 q_{0}\right)^{\frac{1}{6}}}{\left(1+q_{0}\right)^{\frac{2}{3}}}$. By Equations (48) and (71), $A=12.42726525 \times 10^{52} \mathrm{Kg}$. So,

$$
M=\frac{\left(12.42726525 \times 10^{52} \mathrm{Kg}\right)(1+q)^{\frac{2}{3}}}{(1-2 q)^{\frac{1}{6}}}
$$

Next, let

$$
\mu=\frac{\sqrt{3} \sigma}{t_{0}}
$$

Then, by Equation (46), $(1+q)^{\frac{2}{3}}=\frac{6^{\frac{2}{3}} \mathrm{e}^{\frac{2 \mu t}{3}}}{\left(\mathrm{e}^{\mu t}+1\right)^{\frac{4}{3}}}$, and by

Equation (45), $(1-2 q)^{\frac{1}{6}}=\frac{3^{\frac{1}{6}}\left(\mathrm{e}^{\mu t}-1\right)^{\frac{1}{3}}}{\left(\mathrm{e}^{\mu t}+1\right)^{\frac{1}{3}}}$

Then, $M=\frac{\left(3.416825969 \times 10^{53}\right) \mathrm{e}^{\frac{2 \mu t}{3}}}{\left[\left(\mathrm{e}^{\mu t}+1\right)\left(\mathrm{e}^{\mu t}-1\right)\right]^{\frac{1}{3}}} \mathrm{Kg}$. So,
$M_{T}=\frac{\left(3.416825969 \times 10^{53}\right) \mathrm{e}^{\frac{2 \mu T}{3}}}{\left[\left(\mathrm{e}^{\mu T}+1\right)\left(\mathrm{e}^{\mu T}-1\right)\right]^{\frac{1}{3}}} \mathrm{Kg}$.

By Equation (99), this becomes:

$$
\mathrm{e}^{\frac{2 \mu T}{3}}=0.107795938\left(\mathrm{e}^{\mu T}+1\right)\left(\mathrm{e}^{\mu T}-1\right)^{\frac{1}{3}}
$$

By Equation (47), $\sigma=1.487068912$. Also, $t_{0}=H_{0}^{-1}=4.320432 \times 10^{17} \mathrm{~s}$.

So, by Equation (102), $\mu=5.961623536 \times 10^{-18} \mathrm{~s}^{-1}$. For this value of $\mu$, Equation (103) gives:

$$
T=5.545363334 \times 10^{17} \mathrm{~s} \approx 17.884 \times 10^{9} \text { years. }
$$

So, inflexion occurs after $4.184 \times 10^{9}$ years from now.

\subsection{The Reversal of the "Arrow of Time"}

Let in the post-inflexion period, time be denoted by $\tau$ and other scalars have the suffix "c". Also, let $S_{c}$ and $\left(S_{c}+d S_{c}\right)$ be the radii of the 3-sphere, and $H_{c}$ and $\left(H_{c}+d H_{c}\right)$ be the Hubble's Constant therein at times $\tau$ and $(\tau+d \tau)$ respectively. $d \tau=-d t$ will indicate the reversal of the "Arrow of Time".

$S_{c}$ moves from $S_{c}=S_{T}$ to $S_{c}=0$, while $S$ moves from $S=0$ to $S=S_{T}$. So, each value of $S_{c}$ must correspond to a unique value of $S$. So,

$$
S_{c}=S
$$

Also, as the universe contracts,

$$
d S_{c}=-d S \text { (106) }
$$

Next, $S_{c}=S>0$ and $d S_{c}=-d S<0$, and so, $S_{c}+d S_{c}=S-d S<S=S_{c}$. So,

$$
\frac{S_{c}+d S_{c}}{S_{c}}<1
$$

Also,

$$
S_{c}=S>0 \Rightarrow\left|S_{c}\right|=S_{c}
$$

$$
\begin{array}{r}
\text { Now, } S_{c}^{\prime}=H_{c} \Rightarrow \frac{d S_{c}}{\left|S_{c}\right|}=H_{c} d \tau \Rightarrow \frac{d S_{c}}{S_{c}}=H_{c} d \tau \\
\Rightarrow \int \frac{\mathrm{d} S_{c}}{S_{c}}=\int H_{c} \mathrm{~d} \tau \Rightarrow \ln \left|S_{c}\right|=\int H_{c} \mathrm{~d} \tau+A,
\end{array}
$$

where $A$ is the constant of integration. Then, by Equation (108), $\ln S_{c}=\int H_{c} \mathrm{~d} \tau+A$; or,

$$
S_{c}=\mathrm{e}^{A+\int H_{c} \mathrm{~d} \tau}
$$

Then,

$$
S_{c}+d S_{c}=\mathrm{e}^{A+\int\left(H_{c}+d H_{c}\right) \mathrm{d} \tau}
$$

So, by Equation (107),

$$
\mathrm{e}^{\int H_{c} \mathrm{~d} \tau}=\frac{S_{c}+d S_{c}}{S_{c}}<1
$$


Hence,

$$
\int d H_{c} \mathrm{~d} \tau<0
$$

Differentiation of this with respect to $H_{c}$ gives:

$$
d \tau<0
$$

Next, $\tau$ moves from $\tau=T$ to $\tau=0$, while $t$ moves from $t=0$ to $t=T$. So, each value of $\tau$ must correspond to a unique value of $t$. So,

$$
\tau=t
$$

Then, $d \tau=d t>0$, or, $d \tau=-d t<0$. Inequality (109), so, indicates

$$
d \tau=-d t
$$

So, the "Arrow of Time" reverses at the moment of inflexion, i.e. at $t=T$.

\subsection{Matter in the Contracting Universe}

Equation (92) is reproduced below:

$$
t \propto \frac{S}{M}, d t<0
$$

\subsection{Antimatter in the Contracting Universe}

Equation (94) is reproduced below:

$$
t \propto \frac{|m|}{S}, d t<0
$$

\subsection{How Matter Increases in the Contracting Universe}

Reversal of the "Arrow of Time" at $t=T$ reverses the arrows of transformations (95) and (96) as shown below.

$$
\begin{aligned}
& C(-t, m) \rightarrow A(t, M) \\
& D(-t, M) \rightarrow B(t, m)
\end{aligned}
$$

Antimatter decreases in the contracting universe with regressive time $(t)$, as given in relation (113), while matter increases, as shown in relation (112). The disappearing antimatter is converted to matter by the transformation (114), which is part of the reversed inbuilt "Mobius transformation", discussed in Subsection 4.4 and now reversed. As antimatter in the universe is plentiful, the conversion is "continual", and matter increases at the cost of decreasing antimatter.

\subsection{Back to $t=0$}

Relation (112) implies that with regressive time increasing mass of matter effects contraction of the universe, while relation (113) shows that with regressive time decreasing mass of antimatter effects expansion of the universe. With the mass of matter preponderating over that of antimatter, contraction rules over expansion.

As time is regressive, it is back to $t=0$, when by relation (112), $S=0$ and $M=\infty$, and by relation (113), $|m|=0$. Thus $t, S, M$ and $|m|$ are back to their starting values at $t=0$.

\subsection{Symmetry between Matter and Antimatter}

The universe, discussed so far, did not start with symmetry between matter and antimatter, nor this symmetry was there at any instant except at $t=T$. Absence of this symmetry, because of the preponderance of matter over antimatter, denied antimatter a role, as important as the role of matter, in the workings of the universe.

In the next section, the concept of the "Antiworld" is introduced and it is shown that symmetry between matter and antimatter is never disturbed.

\section{The ANTIWORLD}

\subsection{What the ANTIWORLD Is}

The ANTIWORLD is the mirror image of the WORLD. It is like the WORLD except that the fundamental scalar therein, having the subscript "A" in their denotations, bear the following relations with those in the WORLD, having no subscript in their denotations.

$$
\begin{gathered}
t_{A}=-t \\
S_{A}=-S \\
\left|M_{A}\right|=M=\frac{\theta}{|m|} \\
\left|m_{A}\right|=|m|=\frac{\theta}{M}
\end{gathered}
$$

where by Equation(60), $\theta=M|m|$

\subsection{Isolation of Two WORLDS from Each Other}

Time $\left(t_{A}\right)$ in the ANTIWORLD is, by Equation (116) negative. It advances from $t_{A}=0$ to $t_{A}=-\infty$. So, its range is:

$$
0 \geq t_{A} \geq-\infty
$$

On the other hand, time $(t)$ in the WORLD is positive and advances from $t=0$ to $t=\infty$, its range being

$$
0 \leq t \leq \infty
$$

These two ranges show that neither of the WORLDS has in the other's range, and so the WORLD has no past beyond $t=0$, or the ANTIWORLD has no past beyond $t_{A}=0$. This means that except at $t=t_{A}=0$, the two WORLDS are completely isolated from each other by this time barrier. 


\subsection{The ANTIWORLD Is Expanding with the WORLD}

The relation between $H_{A}$, the Hubble's Constant in the ANTIWORLD, and $H$, that in the WORLD, is obtained, using Equations (116) and (117), as is given below.

$$
H_{A}=\left(S_{A}\right)_{t_{A}}=(-S)_{-t}^{\prime}=-S_{-t}^{\prime}
$$

By Equation (77), $-S_{-t}^{\prime}=S_{t}^{\prime}$, and so, $H_{A}=S_{t}^{\prime}$, whence

$$
H_{A}=H
$$

This shows that the ANTIWORLD expands from $S_{A}=0 \quad$ (at $\left.t_{A}=0\right)$ to $S_{A}=-\infty \quad\left(\right.$ at $\left.t_{A}=-\infty\right)$ along with the WORLD, which expands from $S=0$ at $(t=0)$ to $S=\infty$ at $(t=\infty)$.

As at $t_{A}=-t=0, S_{A}=-S=0$ and growth rates of $H_{A}$ and $H$ are equal (vide Equation (122)), $S=S_{t}$ at any instant $t=t$ in the WORLD implies $S_{A}=-S_{t}$ at $t_{A}=-t$ in the ANTIWORLD. That is, at $t_{A}=-t$, the ANTIWORLD is exactly at that stage of expansion, whereat the WORLD is at $t=t$.

\subsection{Antimatter in the Expanding ANTIWORLD}

Equation (94) implies $t_{t}^{\prime}=|m|_{t}^{\prime}-S_{t}^{\prime}$. Then, by Equation

(77), $-t_{-t}^{\prime}=-|m|_{-t}^{\prime}+S_{-t}^{\prime} \Rightarrow(-t)_{-t}^{\prime}=-|m|_{-t}^{\prime}+S_{-t}^{\prime}$

$\Rightarrow-t \propto \frac{S}{|m|}$. Of this, the L.H.S. $\leq 0$ and the R.H.S. $\geq 0$, and so, $-t=\frac{-a S}{|m|}$, where $a(\geq 0)$ is a constant. Then, $-t=\frac{a(-S)}{|m|}$; or, $t_{A}=\frac{a S_{A}}{|m|}$; or, $t_{A}=\frac{a S_{A}}{|m|_{A}}$, whence

$$
t_{A} \propto \frac{S_{A}}{|m|_{A}}
$$

\subsection{Matter in the Expanding ANTIWORLD}

Equation (92) implies $t_{t}^{\prime}=S_{t}^{\prime}-M_{t}^{\prime}$. Then, by Equation (77), $-t_{-t}^{\prime}=-S_{-t}^{\prime}+M_{-t}^{\prime} \Rightarrow(-t)_{-t}^{\prime}=\left(S^{-1}\right)_{-t}^{\prime}+M_{-t}^{\prime}$ $\Rightarrow(-t)_{-t}^{\prime}=\left(\frac{M}{S}\right)_{-t}{ }^{\prime}$, whence $-t \propto \frac{M}{S}$. The L.H.S. of this $<0$, and the R.H.S. $>0$, and so, $-t \propto \frac{-a M}{S}$, where $a(>0)$ is a constant. Then, $-t=\frac{a M}{(-S)}$; or,
$t_{A}=\frac{a M_{A}}{S_{A}}$; whence

$$
t_{A} \propto \frac{M_{A}}{S_{A}}
$$

\subsection{How Matter Increases in the Expanding ANTIWORLD}

Let the points $A, B, C$, and $D$ in the figure of Subsection 4.4 be represented in the ANTIWORLD by $\bar{A}, \bar{B}, \bar{C}$, and $\bar{D}$ respectively. Then, transformation (95) implies:

$$
\bar{A}(-t,-M) \rightarrow \bar{C}(t,-m) \Rightarrow \bar{A}(-t, m) \rightarrow \bar{C}\left(-t_{A}, M\right)
$$

whence,

$$
\bar{A}\left(t_{A}, m_{A}\right) \rightarrow \bar{C}\left(-t_{A}, M_{A}\right)
$$

Next, the transformation (96) implies:

$$
\bar{B}(-t,-m) \rightarrow \bar{D}(t,-M) \Rightarrow \bar{B}(-t, M) \rightarrow \bar{D}(t, m)
$$

whence,

$$
\bar{B}\left(t_{A}, M_{A}\right) \rightarrow \bar{D}\left(-t_{A}, m_{A}\right)
$$

Now, let $\bar{A}, \bar{B}, \bar{C}, \bar{D}$ be the four corners of a rectangular strip of paper. When this strip is given a single twist and the edge $\bar{A} \bar{B}$ is joined with the edge $\bar{C} \bar{D}$ in such a way that $\bar{A}$ and $\bar{B}$ coincide with $\bar{C}$ and $\bar{D}$ respectively, a Mobius strip is formed.

Then, transformations (125) and (126) together constitute a Mobius transformation, operating within the ANTIWORLD. So, under this built-in system of Mobius transformation, anti-particles (in the NTZ) represented by $\bar{A}\left(t_{A}, m_{A}\right)$ undergo conversion to the corresponding particles (in the PTZ) represented by $\bar{C}\left(-t_{A}, M_{A}\right)$. Plenty of antimatter in the ANTIWORLD ensures "continual" conversion of antimatter to matter. Such a scenario supports Equation (60) as well as Equations (123) and (124).

As matter is very rare in the NTZ, transformation (126) rarely occurs.

\subsection{Symmetry between Matter and Antimatter during Expansion}

Equation (123) shows that in the ANTIWORLD, at $t_{A}=0,\left|m_{A}\right|=\infty$. Also, Equation (92) shows that in the WORLD, at $t=0, M=\infty$. So, $\left|m_{A}\right|$ in the ANTIWORLD and $M$ in the WORLD have the same initial values.

Next, $M_{t}^{\prime}=\frac{\frac{\mathrm{d} M}{\mathrm{~d} t}}{|M|}$. So, by Equation (118), 


$$
\begin{gathered}
M_{t}^{\prime}=\frac{\frac{\mathrm{d}\left(\theta\left|m_{A}\right|^{-1}\right)}{\mathrm{d} t}}{\left.|\theta| m_{A}\right|^{-1} \mid}=\frac{\frac{-\theta\left|m_{A}\right|^{-2} \mathrm{~d}\left|m_{A}\right|}{\mathrm{d} t}}{\left(\theta\left|m_{A}\right|^{-1}\right)} \\
=\frac{-\left|m_{A}\right|^{-1} \mathrm{~d}\left|m_{A}\right|}{\mathrm{d} t}=\frac{\frac{-\mathrm{d}\left|m_{A}\right|}{\mathrm{d} t}}{\left|m_{A}\right|}=\frac{\frac{\mathrm{d}\left|m_{A}\right|}{\mathrm{d}(-t)}}{|| m_{A} \|}
\end{gathered}
$$

So, $M_{t}^{\prime}=\left|m_{A}\right|_{-t}^{\prime}$, whence

$$
M_{t}^{\prime}=\left|m_{A}\right|_{t_{A}}^{\prime}
$$

This means that the growth rates of $\left|m_{A}\right|$ in the ANTIWORLD and $M$ in the WORLD are equal.

The initial value and growth rate of $\left|m_{A}\right|$ in the ANTIWORLD being correspondingly equal to those of $M$ in the WORLD implies that the value of $\left|m_{A}\right|$ at any instant $t_{A}=-t$ equals that of $M$ at the corresponding instant $t=t$. That is

$$
\left|m_{A}\right|_{t_{A}}=M_{t}
$$

Next, Equation (124) shows that in the ANTIWORLD at $t_{A}=0, M_{A}=0$. Also, Equation (94) shows that in the WORLD, at $t=0,|m|=0$. That is, $M_{A}$ in the ANTIWORLD and $|m|$ in the WORLD have the same initial values.

Application of Equations (76) and (77) on Equation (127) gives:

$$
-M_{-t}^{\prime}=\left|m_{A}\right|_{-t}^{\prime} \text {; or, }-M_{-t}^{\prime}=-\left|m_{A}\right|_{t}^{\prime} \text {; or, } M_{-t}^{\prime}=\left|m_{A}\right|_{t}^{\prime} \text {; }
$$

or, $\left(M_{A}\right)_{-t}^{\prime}=|m|_{t}^{\prime}$ whence

$$
\left(M_{A}\right)_{t_{A}}^{\prime}=|m|_{t}^{\prime}
$$

This means that growth rates of $M_{A}$ in the ANTIWORLD $|m|$ in the WORLD are equal.

The initial values and growth rates of $M_{A}$ in the ANTIWORLD being correspondingly equal to those of $|M|$ in the WORLD implies that the value of $M_{A}$ at any instant $t_{A}=-t$ equals that of $|m|$ at the corresponding instant $t=t$. That is, $\left(M_{A}\right)_{-t}=|m|_{t}$, whence

$$
\left(M_{A}\right)_{t_{A}}=|m|_{t}
$$

Addition of Equations (128) and (130) gives:

$$
M_{t}+\left(M_{A}\right)_{t_{A}}=\left|m_{A}\right|_{t_{A}}+|m|_{t}
$$

This shows that symmetry between matter and antimatter is maintained throughout the expansion phase of the universe.

\subsection{Roles Played by Antimatter and Matter in the ANTIWORLD}

Equation (123) implies that with progressive time $\left(t_{A}\right)$ decreasing antimatter effects expansion of the ANTIWORLD, while Equation (124) implies that with progressive time increasing matter effects contraction of the ANTIWORLD.

In the WORLD, matter preponderates over antimatter, i.e. $M_{t}>\left|m_{t}\right|$. So, by Equations (128) and (130), this becomes $\left|m_{A}\right|_{t_{A}}>\left(M_{A}\right)_{t_{A}}$, which conveys that in the ANTIWORLD antimatter preponderates over matter. Then, $\left(M_{A}\right)_{t_{A}}<\left|m_{A}\right|_{t_{A}}$ is indicative of the expansion of the ANTIWÓRLD, which will be on the threshold of inflecting into the contraction phase when

$$
\left(M_{A}\right)_{t_{A}}=\left|m_{A}\right|_{t_{A}}
$$

\subsection{The Moment of Inflexion}

It has been seen in Subsection 4.6 that the WORLD inflects into the contraction phase at $t=T$, when

$$
M_{T}=|m|_{T}
$$

Equations (128) and (130) for $t=T$ are respectively

$$
\left|m_{A}\right|_{T_{A}}=M_{T}
$$

and

$$
\left(M_{A}\right)_{T_{A}}=|m|_{T}
$$

Equations (133) and (135) together give:

$$
\left(M_{A}\right)_{T_{A}}=M_{T}
$$

This, with Equation (134), gives:

$$
\left(M_{A}\right)_{T_{A}}=\left|m_{A}\right|_{T_{A}}
$$

This, read with Equation (132), shows that the ANTIWORLD inflects into the contraction phase when $t_{A}=T_{A}=-T$. The mass of antimatter as well as that of matter at $t_{A}=T_{A}$ is given by:

$$
\left(M_{A}\right)_{T_{A}}=\left|m_{A}\right|_{T_{A}}=M_{T}
$$

\subsection{The Reversal of the "Arrow of Time"}

Let in the post-inflexion period, scalars have the suffix " $A C$ ". Also, let $S_{A C}$ and $\left(S_{A C}+d S_{A C}\right)$ be the radii of the 3-sphere, and $H_{A C}$ and $\left(H_{A C}+d H_{A C}\right)$ be the Hubble's Constant therein at times $t_{A C}$ and $\left(t_{A C}+d t_{A C}\right)$ respectively. $d t_{A C}=-d t_{A}$ will indicate the reversal of the "Arrow of Time".

$S_{A C}$ moves from $S_{A C}=S_{A T_{A}}$ to $S_{A C}=0$, while $S_{A}$ moves from $S_{A}=0$ to $S_{A}=S_{A T}$. So, each value of $S_{A C}$ must correspond to a unique value of $S_{A}$. Then,

$$
S_{A C}=S_{A}
$$


Also, as the ANTIWORLD contracts,

$$
d S_{A C}=-d S_{A}
$$

As $S_{A}=-S$ and $d S_{A}=-d S ; S_{A C}=-S$ $d S_{A C}=d S$, and so,

$$
S_{A C}+d S_{A C}=-S+d S=-(S-d S)
$$

Then,

$$
\frac{S_{A C}+d S_{A C}}{S_{A C}}=\frac{-(S-d S)}{-S}=1-\frac{d S}{S},
$$

whence

$$
\frac{S_{A C}+d S_{A C}}{S_{A C}}<1
$$

Next, $S_{A C}^{\prime}=H_{A C} \Rightarrow \frac{\frac{\mathrm{d} S_{A C}}{\mathrm{~d} t_{A C}}}{\left|S_{A C}\right|}=H_{A C}$. As

$S_{A C}=S_{A}=-S<0,\left|S_{A C}\right|=-S_{A C}$. So,

$\frac{\mathrm{d} S_{A C}}{S_{A C}}=-H_{A C} \mathrm{~d} t_{A C} \cdot$ On integration, this yields:

$$
\ln \left|S_{A C}\right|=-\int H_{A C} \mathrm{~d} t_{A C}+B
$$

where $B$ is the constant of integration. Then,

$$
S_{A C}=-\mathrm{e}^{B-\int H_{A C} \mathrm{~d} t_{A C}}
$$

So,

$$
S_{A C}+d S_{A C}=-\mathrm{e}^{B-\int\left(H_{A C}+d H_{A C}\right) \mathrm{dt} t_{A C}}
$$

Therefore,

$$
\frac{S_{A C}+d S_{A C}}{S_{A C}}=\mathrm{e}^{-\int d H_{A C} d_{A C}}
$$

which implies: $\mathrm{e}^{-\int d H_{A C} \mathrm{dt} A C}<1$ or, $\mathrm{e}^{\int d H_{A C} \mathrm{dt} t_{A C}}>1=e^{0}$ whence,

$$
\int d H_{A C} \mathrm{~d} t_{A C}>0
$$

Differentiation of this with respect to $H_{A C}$ yields:

$$
d t_{A C}>0
$$

Now, $t_{A C}$ moves from $t_{A C}=T_{A}$ to $t_{A C}=0$, while $t_{A}$ moves from $t_{A}=0$ to $t_{A}=T_{A}$. So, each value of $t_{A C}$ must correspond to a unique value of $t_{A}$. So,

$$
t_{A C}=t_{A}
$$

Then, either $d t_{A C}=d t_{A}$; or, $d t_{A C}=-d t_{A}$.

In the former case, $d t_{A C}=d t_{A}=-d t<0$, which contradicts Equation (140). So,

$$
d t_{A C}=-d t_{A}
$$

This indicates that there is the reversal of the "Arrow of Time" at $t_{A C}=T_{A}$.

\subsection{Antimatter in the Contracting ANTIWORLD:}

Equation (123) is reproduced below:

$$
t_{A} \propto \frac{S_{A}}{|m|_{A}}, \quad d t_{A}>0
$$

\subsection{Matter in the Contracting ANTIWORLD}

Equation (124) is reproduced below:

$$
t_{A} \propto \frac{M_{A}}{S_{A}}, \quad d t_{A}>0
$$

\subsection{How Antimatter Increases in the Contracting ANTIWORLD}

With the reversal of the "Arrow of Time", the arrow of the transformation (125) gets reversed as follows:

$$
\bar{C}\left(-t_{A}, M_{A}\right) \rightarrow \bar{A}\left(t_{A}, m_{A}\right)
$$

Matter decreases in the contracting ANTIWORLD with regressive time $\left(t_{A}\right)$, as given in relation (146), while antimatter increases, as shown in relation (145). The disappearing matter is converted to antimatter by the transformation (147), which is a part of reversed in-built "Mobius transformation" discussed in Subsection 5.6 and now reversed.

As matter in the ANTIWORLD is plentiful, the conversion is continual, and antimatter increases at the cost of decreasing matter.

\subsection{Back to $t_{A}=0$}

Relation (145) implies that with regressive time increasing mass of antimatter effects contraction of the ANTIWORLD, while relation (146) shows that with regressive time decreasing mass of matter effects its expansion. With the mass of antimatter preponderating over that of matter, contraction rules over expansion.

As time is regressive, it is back to $t_{A}=0$, when, by relation (145), $S_{A}=0$ and $\left|m_{A}\right|=\infty$, and by relation (146), $M_{A}=0$. Thus, $t_{A}, S_{A},\left|m_{A}\right|$ and $M_{A}$ are back to their starting values at $t_{A}=0$.

\subsection{Symmetry between Matter and Antimatter during Contraction Phase}

Equation (145) shows that in the ANTIWORLD at $t_{A}=0,\left|m_{A}\right|=\infty$. Also, Equation (112) shows in the WORLD at $t=0, M=\infty$. So, $\left|m_{A}\right|$ in the ANTIWORLD and $M$ in the WORLD have the same initial values.

Next, Equation (127) shows that $\left|m_{A}\right|_{t_{A}}^{\prime}=M_{t}^{\prime}$, i.e., the growth rates of $\left|m_{A}\right|$ in the ANTIWORLD and $M$ in the WORLD are equal. 
The initial value and growth rate of $m_{A}$ being correspondingly equal to those of $M$ implies that the value of $\left|m_{A}\right|$ at any instant $t_{A}=-t$ equals that of $M$ at the corresponding instant $t=t$. That is,

$$
\left|m_{A}\right|_{t_{A}}=M_{t}
$$

Next, Equation (146) shows that at $t_{A}=0, M_{A}=0$. Also, Equation (113) shows that at $t=0,|m|=0$. That is, $M_{A}$ in the ANTIWORLD and $|m|$ in the WORLD have the same initial value.

Application of Equation (77) on Equation (127) gives:

$$
-\left|m_{A}\right|_{-t_{A}}{ }^{\prime}=-M_{-t}^{\prime} \text {; or, }-\left|m_{A}\right|_{t}^{\prime}=-M_{t_{A}}^{\prime}, \Rightarrow\left|m_{A}\right|_{t}^{\prime}=M_{t_{A}}^{\prime}
$$

whence

$$
|m|_{t}^{\prime}=\left(M_{A}\right)_{t_{A}}^{\prime}
$$

The initial value and growth rate of $|m|$ in the WORLD being correspondingly equal to those of $M_{A}$ in the ANTIWORLD implies that the value of $M_{A}$ at any instant $t_{A}=-t$ equals that of $|m|$ at the corresponding instant $t=t$. That is $\left(M_{A}\right)_{-t}=|m|_{t}$, whence

$$
|m|_{t}=\left(M_{A}\right)_{t_{A}}
$$

Addition of Equations (148) and (150) gives:

$$
M_{t}+\left(M_{A}\right)_{t_{A}}=|m|_{t}+\left|m_{A}\right|_{t_{A}}
$$

This shows that the symmetry between matter and antimatter is maintained throughout the contraction phase of the universe.

It has been proved in subsection 5.7 that this symmetry is maintained throughout the expansion phase of the universe. So, this symmetry is absolute in the universe.

\section{The Big Bang}

At $t=0$ in the WORLD at the end of the contraction phase, by Equation (112), $S=0$ and $M=\infty$, and by Equation (113), $|m|=0$.

At $t_{A}=-t=0$ in the ANTIWORLD at the end of the contraction phase, by Equation (145), $S_{A}=0,\left|m_{A}\right|=\infty$, and by Equation (146), $M_{A}=0$.

At $t=t_{A}=0$, the time-barrier disappears for an instant, when an infinite mass of antimatter of the ANTIWORLD meets an infinite mass of matter of the WORLD at a point $\left(S=S_{A}=0\right)$, triggering what is known as the BIG BANG, the violent encounter between infinite quantities of matter and antimatter.

Instantaneously with the BIG BANG, the WORLD and the ANTIWORLD are thrown apart by an infinite repulsive force. The force is repulsive because the product, $M m_{A}$, is negative, is infinite as each of $M$ and $m_{A}$ is infinite and the distance between the two worlds is zero.

\section{Conclusion}

This work presents a complete oscillatory cosmological scenario. It is proposed that the universe comprises a WORLD and an ANTIWORLD. Matter/antimatter operates in the positive time zone (PTZ)/negative time zone (NTZ) in both. Antimatter is rare in the WORLD, because its entry in the PTZ is forbidden by a time-barrier. It is assumed to have a negative mass and is abundant in the NTZ of the WORLD. It is successfully shown that decrease in matter/antimatter causes the accelerative expansion of the WORLD/ANTIWORLD. For the Friedman's flat universe with the cosmological constant a theoretical evaluation of the deceleration parameter is $q_{0}=-0.606$ which is in agreement with $q_{0}=-0.6$ by assuming General Relativity or by using Supernova Ia data. It is proposed that a built in transformation in the WORLD/ANTIWORLD converts matter/antimatter (in the PTZ/NTZ) to antimatter/matter (in the NTZ/PTZ). The present study has also revealed that in the expanding WORLD/ANTIWORLD, decreasing matter/antimatter preponderates over increasing antimatter/matter till mass of matter and that of antimatter become equal. Then the WORLD/ANTIWORLD starts contracting, and the "Arrow of Time" reverses. Time moves back to $t=0$, when mass of matter/antimatter of the WORLD/ANTIWORLD is infinite. The WORLD/ANTIWORLD with infinite mass of matter/antimatter meets at $t=0$, when the timebarrier lifts for an instant, to trigger the BIG BANG, the violent encounter between matter and antimatter. It has also been established that the symmetry between matter and antimatter in the universe is maintained throughout.

\section{Acknowledgements}

SM wishes to acknowledge that the originality of this paper rests solely with SKBT. The ideas put forth in the paper have their foundation based mainly on these books [10-15].

\section{REFERENCES}

[1] A. Einstein, “The Meaning of Relativity,” Oxford and IBH Publishing Co., Oxford, 1965.

[2] J. V. Narlikar, "Introduction to Cosmology," Cambridge University Press, New York, 2002.

[3] V. Rydnik, “ABC of Quantum Mechanics,” Mir Publishers, Moscow, 1968.

[4] S. Hawking, “A Brief History of Time,” Bantam Books, 1988.

[5] A. J. van de Ven Antoine, “Antimatter with Negative Mass as a Candidate for Dark Energy," The Dark Universe Conference, Heidelberg, 4-7 October 2011.

[6] A. J. van de Ven Antoine, "A Space-Time Formalism with Negative Mass to describe Antimatter and Dark Energy.” 
[7] H. Bondi, "Negative Mass in General Relativity," Reviews of Modern Physics, Vol. 29, No. 3, 1957, pp. 423-428. doi:10.1103/RevModPhys.29.423

[8] F. Capra, “The Tao of Physics,” Wildwoodc House, University Press Oxford, Oxford, 1975.

[9] S. Hawking and R. Penrose, "The Nature of Space and Time,” Oxford University Press, Cambridge, 1997.

[10] V. A. Ugarov, “Special Theory of Relativity,” Mir Publishers, Moscow, 1979.

[11] D. Griffiths, "Introduction to Elementary Particles,” John Wiley and Sons, New York, 1987. doi:10.1002/9783527618460

[12] S. W. Hawking and G. F. R. Ellis, “The Large Scale Structure of Space-Time,” Cambridge University Press, Cambridge, 1973. doi:10.1017/CBO9780511524646

[13] P. S. Joshi, "Global Aspects in Gravitation and Cosmology,” Oxford University Press, Cambridge, 1979.

[14] R. M. Wald, “General Relativity,” University of Chicago Press, Chicago, 1984. doi:10.7208/chicago/9780226870373.001.0001

[15] M. Bartusiak, “Archives of the Universe,” Pantheon Books, New York, 2004. 